\title{
FORCED OSCILLATION AND HIGHER HARMONIC DETECTION IN AN INTEGRATED CMOS-MEMS SCANNING PROBE MICROSCOPE
}

\author{
N. Sarkar ${ }^{1 *}$, R. R. Mansour ${ }^{l}$ and K. Trainor ${ }^{l}$ \\ ${ }^{1}$ University of Waterloo, Waterloo, Ontario, Canada
}

\begin{abstract}
We present the first imaging results obtained with a MEMS scanning probe microscope (SPM) that does not require external scanning hardware, and uses forced oscillation and higherharmonic detection to eliminate thermal coupling. This SPM supports multiple modes of operation including contact AFM (atomic force microscopy), intermittent-contact AFM, and thermal profiling. The focus of this paper is on the intermittent-contact mode, in which we are interested in measuring topology while suppressing thermal effects. The method has been implemented with three SPM-on-a-chip designs, and consistently improves resolution.
\end{abstract}

\section{INTRODUCTION}

\section{Motivation}

The goal of this work is to miniaturize bulky piezoelectric positioning systems that suffer from thermal drift, poor vibration immunity, and creep by replacing them with MEMS electrothermal actuators with integrated position sensors. We also replace laserbased deflection sensing with integrated polysilicon strain sensors. This permits array implementations, and dramatically lowers the cost of SPM's. One of the challenges in the development of our CMOS-MEMS SPM's is that interaction between electrical, thermal and mechanical domains obscures the desired measurement of tip-sample interaction. In particular, the piezoresistive strain sensors that are used to measure tip-sample interaction forces have a large TCR (temperature coefficient of resistivity). This problem is exacerbated by the use of thermal actuation, which results in a large range of operating temperatures. In addition, high temperatures result in unwanted actuation of the strain sensors and this contributes to the tip-sample interaction signal. We therefore seek a method to extract only the tip-sample interaction component of a signal that contains various thermally induced parasitics.

\section{Review}

In [1], we reported the first CMOS-MEMS contact-mode AFM with integrated actuation and position/force sensing in 3-D. The resolution was compromised by thermal coupling from actuation because of the high temperature coefficient of resistivity (TCR) of the polysilicon strain sensors. Frequent thermal calibration and post-processing of the data was required in order to extract the sample topology from the cantilever signal.

The first micromachined STM consisted of a piezoelectric bimorph with patterned electrodes [2]. Another STM was fabricated in single crystal silicon [3]. These works demonstrated open-loop 3-axis positioning only, with no force-sensing capability. More recently the co-fabrication of control electronics with an array of single-axis cantilevers for AFM was demonstrated [4]. Another example of a single-axis cantilever with integrated position sensing is the Akiyama probe [5]. In [6], a thermal profiler with integrated 1-D actuation was presented. Our work was the first to include 3-axis positioning, strain sensors, and position sensors (all the mechanical components required for an SPM to acquire an image) in a single device.

\section{Detection Method}

Figure 1 is a system diagram of the method that is used to separate the tip-sample interaction component from the overall signal. A small-signal sinusoidal input with a DC offset $V$ is applied to the Z-actuator. Heat from actuation couples to the piezoresistors, producing effects in the electrical (due to TCR) and mechanical (due to stress induced by the thermal bimorph effect) domains. The balanced cantilevers are biased with a DC voltage that is also applied to an off-chip trimming potentiometer. This balanced configuration rejects a fraction of the parasitic thermal coupling, but several asymmetries in the design remain and contribute thermal parasitics.

We focus on the case in which the tip is in intermittent contact with the sample due to the periodic excitation of the Z-actuator. At the point of contact, abrupt changes occur in the mechanical and thermal boundary conditions at the tip, generating higher harmonic terms. An instrumentation amplifier follows the bridge, and its output is demodulated using a pair of off-the-shelf modulatordemodulator IC's. The demodulation frequency is chosen at a harmonic that is above the thermal cutoff frequency. We hold the harmonic power constant by adjusting the Z-offset voltage (hence tracking topology) while scanning laterally in $\mathrm{X}$ and $\mathrm{Y}$. In this paper, we do not present imaging results using the phase component of the signal.

This configuration rejects thermal parasitics from the TCR of the piezoresistors. Since we maintain a constant tip-height separation during the scan, the controller intrinsically compensates for the thermal-mechanical coupling between the lateral and

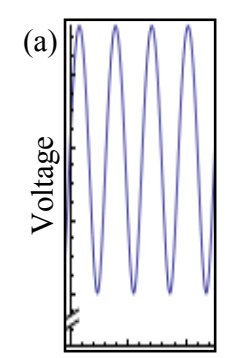

time

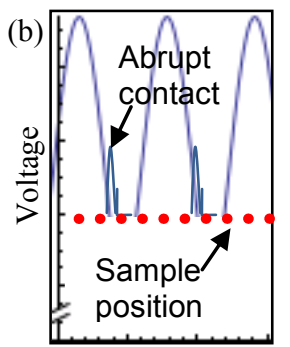

time

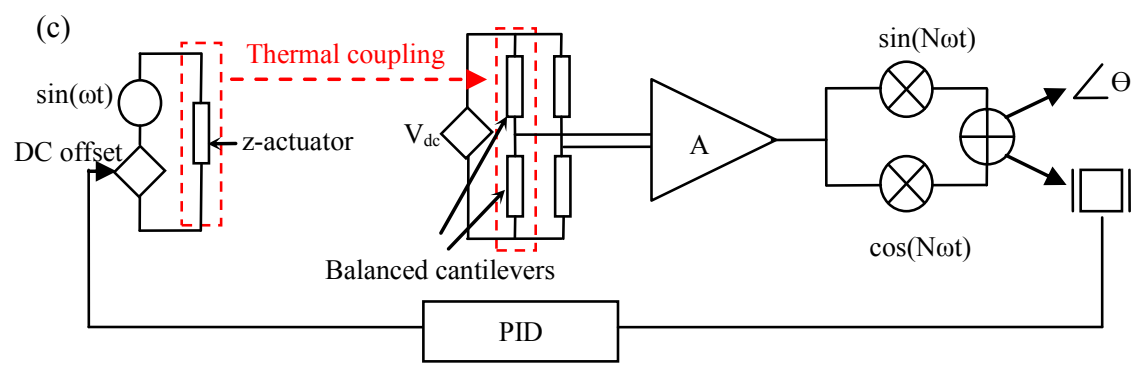

Figure 1: (a) z-actuator driving signal is sinusoidal with a DC offset, (b) piezoresistive signal is clipped by intermittent contact with the sample, and (c) diagram of the measurement setup. MEMS devices are enclosed in red. The z-actuator is oscillated at the fundamental frequency, and a DC offset is used to control the tip-sample gap to control the measured power in the $N^{\text {th }}$ harmonic. 
vertical actuators of the device as well. The result is an image which is tilted on a plane that represents the average temperature of the lateral scanners. This tilt is easily removed with a linear coordinate transformation.

\section{DEVICE DESIGN}

\section{Process}

CMOS-MEMS processes were introduced in the 1980's and the field has grown substantially over the past two decades. [7] These processes are well-suited to integrated SPM device fabrication because they allow for complex signal routing, various types of actuation and sensing methods, and integrated electronics. Some examples of CMOS cantilever sensor systems that exploit the various material properties available in CMOS-MEMS processes are discussed in [8].

Our fabrication process is similar to the post CMOS-MEMS processes, with the optional insertion of two additional maskless process steps. First, grooves are diced or laser-etched into the backside of the wafer to suspend cantilevers over the die edge upon release. The second step removes polymer buildup that occurs during the $\mathrm{SiO}_{2}$ etch using EKC.

There are several process features that are advantageous in the design of CMOS-MEMS SPM's:

In the electrical domain, the designer can choose between conductive (Al), insulating $\left(\mathrm{SiO}_{2}\right)$, and semiconducting (polysilicon) materials. This allows for complex routing of signals in a monolithic beam. It also allows for the judicious placement of resistive heat sources and temperature sensors. The temperature coefficient of resistivity of polysilicon materials can be exploited to measure local temperatures, a feature that is used in our work.

The thermal conductivity of $\mathrm{Al}$ is $191 \mathrm{Wm}^{-1} \mathrm{~K}^{-1}$ while that of $\mathrm{SiO}_{2}$ is $1.4 \mathrm{Wm}^{-1} \mathrm{~K}^{-1}$. This enables the design of thermally isolating members that can be used to separate heat sources from nearby regions that are mechanically and electrically interconnected. Another feature is that the polysilicon-metal contacts in CMOSMEMS processes are thermocouples that can be used as local temperature sensors.

In the mechanical domain, the thermal expansion coefficients (TCE) of $\mathrm{Al}$ and $\mathrm{SiO}_{2}$ are disparate as well. The TCE of $\mathrm{Al}$ is $14.6+/-3.0 \times 10^{-6} \mathrm{~K}^{-1}$ and the TCE of $\mathrm{SiO}_{2}$ is $0.4-1.0 \times 10^{-6} \mathrm{~K}^{-1}$. This feature is desirable in the design of lateral and vertical electrothermal bimorph actuators. Further discussion of the mechanical material properties and how they relate to electrothermal actuator design can be found in [9].

Although a wide variety of material properties are available to the designer, they are seldom optimized to improve SPM performance. For instance, the performance of CMOS electronics improves with low TCR whereas the design of temperature-based position sensors improves with high TCR. Another example is that piezoresistive cantilevers benefit from a high gauge factor, while it is desirable to suppress strain effects in CMOS.

\section{Geometry}

Examples of device geometries are shown in figures 2-4. All three devices contain a balanced pair of piezoresistive cantilevers and various lateral actuator layouts. In figure 2, the lateral actuators are orthogonally arranged and coupled using flexures in an arrangement that cancels the torque applied to the central shuttle. This geometry requires a sample to be lowered onto the chip surface, making it difficult to observe the tip-sample region under an optical microscope. In addition, the flexures are compliant in the z-direction, reducing the strain that is captured by the piezoresistors.

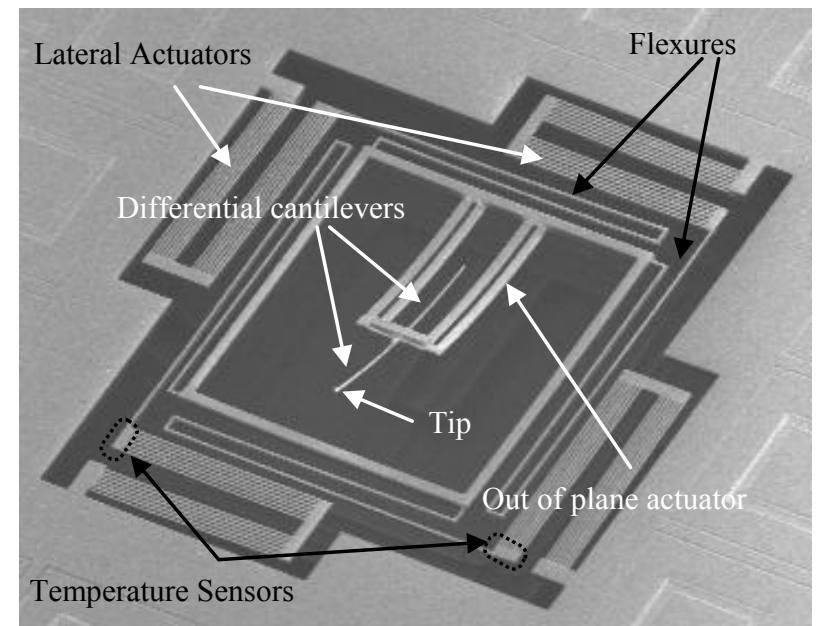

Figure 2: Orthogonally arranged lateral actuators are used to laterally scan the central shuttle which houses the vertical actuator and a balanced pair of cantilevers.

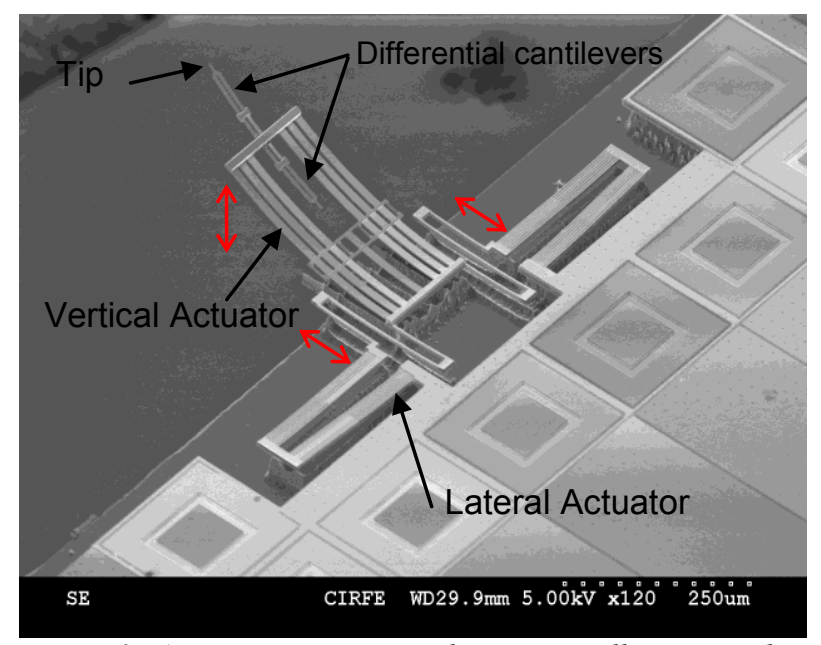

Figure 3: Actuators are arranged symmetrically to scan the tip in spherical coordinates. The tip is suspended over the edge of the chip for observation under an optical microscope.

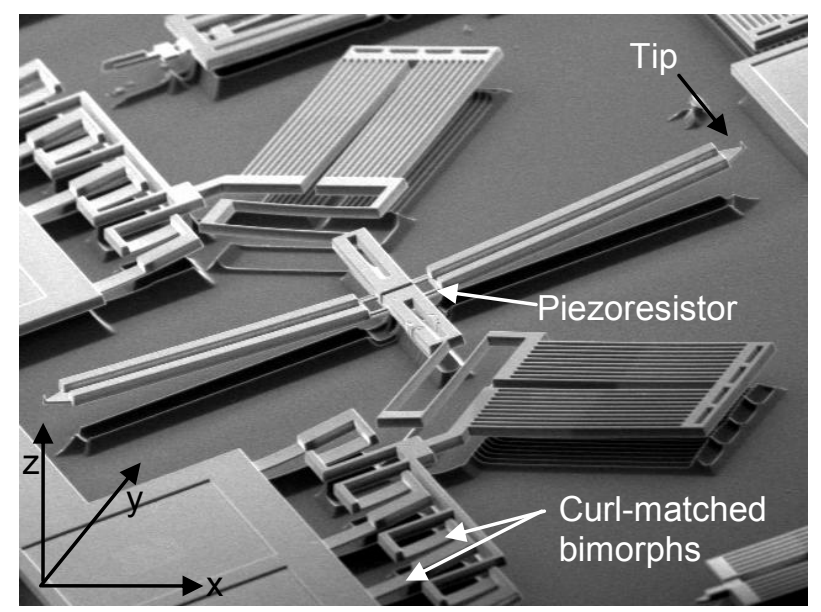

Figure 4: Perspective view of a Cartesian coordinate scanner with an extended cantilever. Z-direction actuation is achieved with a curl-matched bimorph design. 
In figure 3 , the cantilever extends $\sim 300 \mathrm{~m}$ past the edge of the die. This geometry permits observation of the tip-sample region while imaging. Another feature of this geometry is that the average temperature during a lateral scan line can be held constant. This is achieved by applying a DC bias and a differential AC signal to the left and right actuators. A non-linear inversion is applied to the sum of the DC and AC signals in order to compensate for the $\mathrm{V}^{2}$ dependence of the actuators' position and temperature. The result is a spherical coordinate scan $(r, \varphi, \theta)$ in which the angle $\varphi$ is related to the difference between the left and right actuator deflections, the radius $r$ is proportional to the average of the left and right actuator deflectionsand the elevation $\theta$ is provided by the out-of-plane bimorph.

In figure 3 , the cantilever is designed to extend $\sim 200 \mathrm{~m}$ past the die edge. The chip presented here has not yet been singulated. This geometry scans in Cartesian coordinates; the Z-actuator is curl-matched to cancel arcuate motion that is usually encountered with out-of-plane bimorph designs.

Figure 5 is a lumped element model (of the device in Figure 2) that considers effects in the electrical, thermal and mechanical domains. The model follows conventions from [10] and is founded on the work described in [11]. Of particular interest in the design of multi degree-of-freedom SPM scanners is the nature of the interconnection between the various actuators in the system. For example, in the thermal domain, the $\mathrm{X}$ and $\mathrm{Y}$ actuators have a direct conductive path to thermal ground, whereas the $\mathrm{Z}$ actuator and balanced cantilevers do not (neglecting air). The non-linearity of the tip-sample interaction is modeled using a voltage-controlled switch, with a threshold corresponding to the sample height.

\section{MEASUREMENTS}

The separation of the tip-sample force component of the signal from the parasitic thermo-electric and thermo-mechanical components is clearly visible in the frequency domain. Figure 6 presents the frequency spectrum of the cantilever signal when there is no sample present. The a.c. component of the driving signal is a square-root-sinusoid at $200 \mathrm{~Hz}$, which is squared by the actuator's transfer function to obtain the single peak visible in the plot. In figure 7 , the tip enters intermittent contact with the sample and effectively clips the signal from the balanced piezoresistors. Higher harmonics that are well beyond the thermal cutoff frequency appear in the frequency spectrum, and are used to control the tip-sample separation in practice. The plots were obtained with a Zurich Instruments lock-in amplifier.

Images of a calibration grating were obtained using the device in figure 2. The calibration grating geometry is inset in figure 8 . These images were obtained without the use of a lock-in amplifier, and with no post-processing other than tilt-compensation implemented in realtime in the Labview envirionment.

This approach has several advantages over the contact mode implemented in [1]. Higher-harmonic detection methods are capable of revealing material properties beyond topology [12].

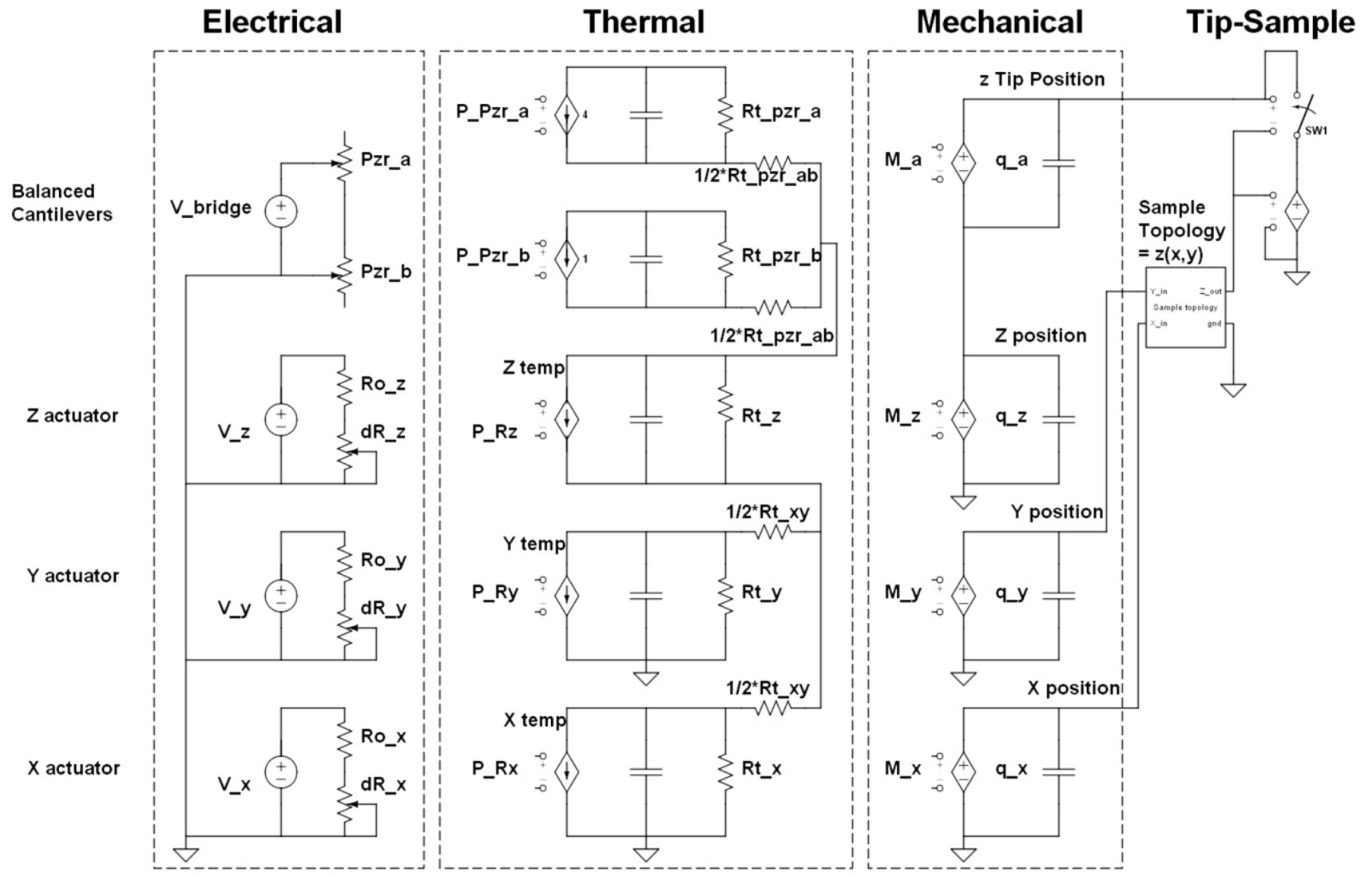

Figure 5: Lumped element model of a CMOS-MEMS SPM in the electrical, thermal and mechanical domains. In the electrical domain, Ro_*labels are electrical resistances and $d R_{-}^{*}$ labels account for the TCR. In the thermal domain, $P_{-}^{*}$ labels represent electrical power converted to heat, and $R t_{-}^{*}$ labels are thermal resistances. In the mechanical domain, $M_{-}^{*}$ labels correspond to thermal moments and $q_{-}{ }^{*}$ labels represent the charge stored on capacitors (analogous to the displacement of beams). The non-linear tip-sample interaction is modeled with a voltage-controlled switch that "shorts" the tip position to the sample's z-height. 


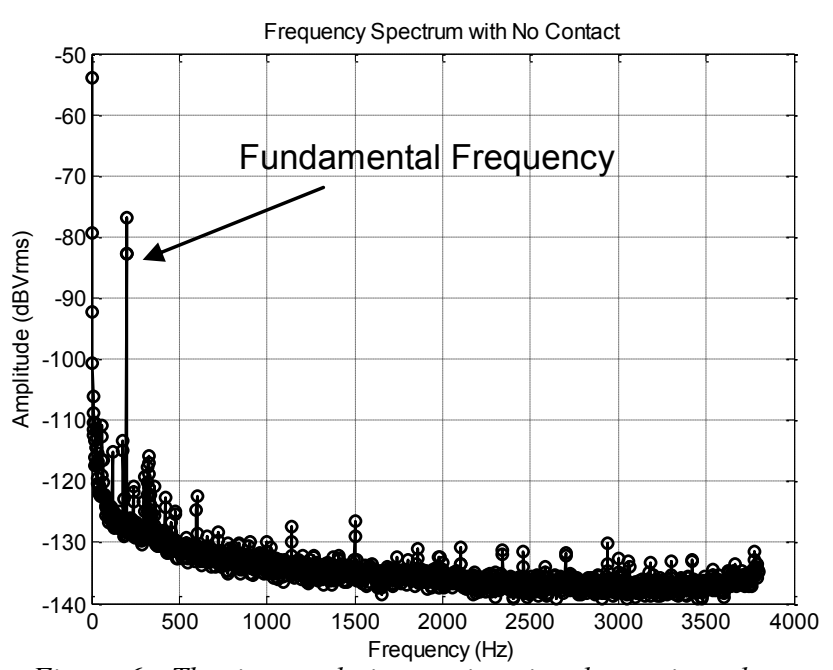

Figure 6: The tip sample-interaction signal contains a large parasitic thermal component at the fundamental frequency.

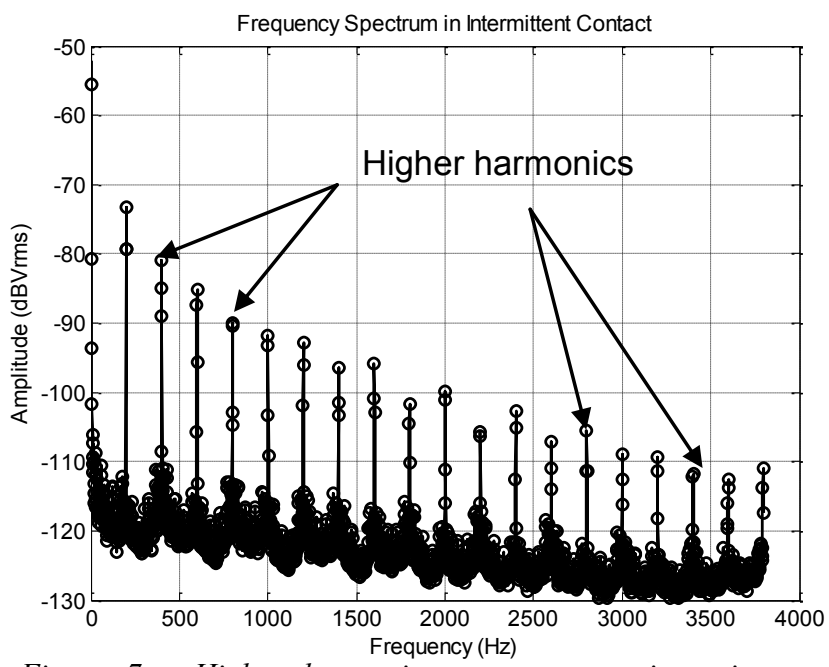

Figure 7: Higher harmonics appear upon intermittent contact.
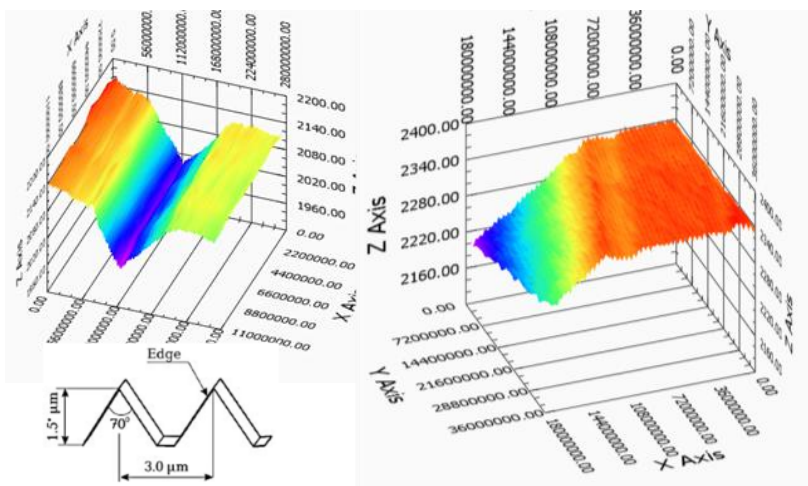

Figure 8: Images of an AFM calibration grating with an inset describing the geometry

This method also permits operation in liquids and does not require Q-control to improve imaging bandwidth [13]. Mechanical modes of the cantilever need not be matched to the harmonic frequencies. The mixer circuit is simple and inexpensive since the cantilever is not in resonance. Tip-sample forces are limited by reducing the higher-harmonic amplitude or adjusting phase to operate in the attractive regime. We have observed that small-amplitude oscillation also eliminates lateral stiction with the sample, which is an issue in the contact mode.

\section{CONCLUSION}

The integration of scanning probe microscopes on-chip has the potential to reduce their cost as well as improve their performance when compared to the state-of-the-art. Electrothermal actuation provides low-voltage (CMOS-compatible) and large scan range; however it introduces parasitics to the tip-sample interaction signal. Higher harmonic detection has been demonstrated as a method to mitigate this issue.

\section{REFERENCES}

[1] N. Sarkar et. al., "CMOS-MEMS Atomic Force Microscope," TRANSDUCERS 2011, pp. 2610-2613.

[2] Akamine, S. et al.,"Microfabricated Scanning Tunneling Microscope," IEEE Electron Device Letters, V. 10, No 11, 1989.

[3] $\mathrm{Xu}, \mathrm{Y}$. et al. "Integrated micro-scanning tunneling microscope," Appl. Phys. Lett. 67 (16), 1995.

[4] D. Barrettino, et al., "CMOS Monolithic Atomic Force Microscope," 2004 Symposium On VLS1 Circuits Digest of Technical Papers, pp. 306-309.

[5] http://www.akiyamaprobe.com

[6] Gianchandani, Y. B. et al, "A Silicon Micromachined Scanning Thermal Profiler with Integrated Elements for Sensing and Actuation," IEEE Trans. Elec. Dev., v 44, no 11, pp 1857-1868.

[7] Baltes, Brand, Fedder, Hierold, Korvink, Tabata, "CMOSMEMS," volume 2, Wiley, 2004.

[8] Lange, Brand, Baltes, "CMOS Cantilever Sensor Systems,' Springer, 2002.

[9] Gilgunn, P.J.et al., "CMOS-MEMS Lateral Electrothermal Actuators," J. Microelectromech. Syst., 17 (2006), pp. 103 114.

[10] Senturia, S.D., "Microsystem Design," Kluwer, 2001.

[11] Todd, S. et. al., "An electrothermomechanical lumped element model of an electrothermal bimorph actuator," J. Microelectromech. Syst., 17, (2008), pp. 213-225.

[12] Sahin, O. et al, Springer Handbook of Nanotechnology, p.717 , 2007.

[13] "High-speed tapping mode imaging with active Q-control for AFM,” T. Sulchek et. al., Appl. Phys. Lett. 76 (1473), 2000.

\section{ACKNOWLEDGEMENTS}

The authors would like to acknowledge the support of TowerJAZZ for their donation of fabrication area, and the Canadian Microelectronics Corporation. This work is supported by DARPA, SPAWARSYSCEN-SD, and the Texas ETF under contract N66001-08-C-2040.

\section{CONTACT}

*N. Sarkar, tel: +1-519-888-4567; nsarkar@uwaterloo.ca 